\title{
Indicateurs physiologiques pour le screening de génotypes de tournesol tolérants aux basses températures associées au semis précoce
}

\author{
Clémentine ALLINNE ${ }^{1}$ \\ Pierre MAURY ${ }^{1}$ \\ Philippe DEBAEKE ${ }^{2}$ \\ Ahmad SARRAFI ${ }^{3}$ \\ Philippe GRIEU ${ }^{1}$ \\ ${ }^{1}$ Université de Toulouse, \\ INPT, UMR AGIR, ENSAT, \\ 31326 Castanet Tolosan, France \\ <maury@ensat.fr> \\ 2 INRA, UMR AGIR, \\ 31326 Castanet Tolosan, France \\ ${ }^{3}$ Université de Toulouse, \\ INPT, IFR 40, \\ Laboratoire SP2, \\ ENSAT, 31326 Castanet Tolosan, France
}

\begin{abstract}
Sunflower is a summer crop often subjected to water stress, which could reduce substantially the yield. Early sowing is one of the crop management strategies to escape the drought stress mainly during flowering. Sowing one or two months earlier may lead to place the seeds and seedlings growth in conditions of low temperature. Low temperature exposure has consequences for most biological processes. It initiates also a number of physiological changes which can lead the plant to be more cold tolerant. Physiological traits commonly used to underlying cold tolerance, as well as traits associated with growth capacity at low temperature, are presented and discussed in the article. The most important ones need to be evaluated in sunflower to explore genetic variability.
\end{abstract}

Key words: early sowing, Helianthus annuus L., low temperature, sunflower, physiological traits, cold tolerance
Différentes stratégies agronomiques et génétiques ont été envisagées pour améliorer la productivité du tournesol en présence de contraintes thermiques et hydriques. Un programme a notamment été développé dans le cadre du projet «Tournesol 2010 » afin d'évaluer les possibilités d'une anticipation des dates de semis dans le but d'accroître la productivité et d'esquiver la contrainte hydrique. Le semis précoce du tournesol d'un à deux mois par rapport à la période habituelle (avril dans le sud-ouest de la France) induit une baisse significative de la température au moment du semis et pendant les premiers stades de la croissance végétative. La mise en œuvre de cette conduite culturale et son évaluation reposent sur I'utilisation de variétés spécifiquement adaptées aux basses températures. La caractérisation de génotypes de tournesol adaptés à des conditions de basses températures en début de cycle nécessite $d$ 'analyser les effets de ces dernières sur les processus physiologiques associés à la vigueur à la levée et à la tolérance au froid afin de disposer d'indicateurs permettant de caractériser une large gamme de matériels génétiques (population de lignées en ségrégation, variétés...).

Après avoir présenté les principaux processus affectés par les basses températures, différents indicateurs physiologiques seront proposés au regard de cette analyse bibliographique pour explorer des différences génotypiques d'adaptation au froid chez le tournesol.

\section{Processus physiologiques conditionnant la levée du tournesol à basse température}

La levée au champ dépend de l'interaction entre la graine et son milieu, incluant de nombreux facteurs environnementaux dont la température, l'humidité, la structure et la composition du sol (Hatfield et Egli, 1974). La phase hétérotrophique se décompose en deux phases physiologiques distinctes: les processus de germination et d'élongation de l'hypocotyle.

La germination a lieu en trois temps : l'imbibition qui correspond à l'hydratation de la semence en présence d'eau, la germination stricto sensu et la phase de croissance durant laquelle débute l'allongement de la radicule. La germination stricto sensu et la croissance sont des processus qui ne mettent pas en jeu les mêmes mécanismes régulateurs, la germination stricto sensu est beaucoup plus sensible aux facteurs du milieu comme la température (Côme and Corbineau, 1998).
La température intervient directement en agissant sur la vitesse des réactions biochimiques dans la graine, une augmentation de température accélère la germination. À basse température, le processus de germination est plus lent qu'à température optimale en raison des nombreux processus métaboliques impliquant des réactions enzymatiques réduites avec le froid. La courbe de réponse de vitesse de germination en fonction de la température permet de déterminer trois températures caractéristiques de chaque espèce et/ou variété : la température minimale de germination ou température de base $(\mathrm{Tb})$ en dessous de laquelle la vitesse de germination est nulle, la température optimale à laquelle la vitesse de germination est la plus élevée et la température maximale à partir de laquelle la vitesse de germination est nulle. Ces trois températures sont appelées températures cardinales pour la germination. La disponibilité en eau va également influencer la vitesse de germination. Un déficit hydrique va augmenter le temps nécessaire à la germination, et il existe pour chaque espèce une valeur seuil ou «potentiel hydrique de base » en dessous de laquelle la germination n'a plus lieu (Durr et al., 2001). L'action combinée du potentiel hydrique et de la température a conduit au concept de temps hydrothermique (Alvarado et 
Bradford, 2002). Mais sans contrainte hydrique, le modèle temps thermique est suffisant pour estimer la Tb de germination (Benech-Arnold et Sanchez, 1995).

Comme pour la phase de croissance de la radicule, la température influence la vitesse d'élongation de l'hypocotyle, et il existe une température minimale de croissance de I'hypocotyle. Cette Tb est très variable entre espèces, elle a été par exemple estimée à $9{ }^{\circ} \mathrm{C}$ chez le maïs (Blacklow, 1972) et à $1,4^{\circ} \mathrm{C}$ chez l'oignon (Wheeler et Ellis, 1991). Ce caractère présente également une variabilité intraspécifique comme cela a été montré chez la luzerne avec des valeurs variant entre 5,5 à $7,5^{\circ} \mathrm{C}$ selon les génotypes (Brunel et al., 2009). Chez le tournesol, la variabilité génétique de composition des graines pourrait contribuer à modifier la réponse des processus de germination et d'élongation à la température. À notre connaissance, les températures de base de germination et d'élongation de I'hypocotyle ne sont pas rapportées dans la littérature pour le tournesol.

\section{Impact des basses températures sur le fonctionnement photosynthétique des plantes}

La photosynthèse se déroule en deux phases distinctes: la phase photochimique durant laquelle l'énergie lumineuse est captée puis transformée en ATP et pouvoir réducteur et le cycle de Calvin qui correspond à la phase de fixation du carbone et permet la synthèse de sucre. Les basses températures affectent la phase photochimique en réduisant la capacité de transfert des électrons via la chaîne de transporteur d'électrons et réduisent également la fixation du carbone qui s'effectue par le biais de réactions biochimiques dont l'activité diminue avec la température (Ensminger et al., 2006). Cette altération du fonctionnement photochimique peut conduire à l'accumulation de ROS (reactive oxygen species), molécules toxiques pour la plante. Si la quantité de ROS formée est supérieure à la quantité détruite via le système antioxydant, les ROS occasionnent des dégâts au sein de la cellule en dégradant les pigments, les membranes des thylakoïdes, les protéines et les enzymes clés du métabolisme carboné. Ces dommages, plus ou moins rapidement réversibles, peuvent accentuer les phénomènes de photo-inhibition (Allen et Ort, 2001).

Il existe plusieurs types de réponses de la plante pour limiter la photo-inhibition en condition de basses températures. À l'échelle des photosystèmes, l'ajustement de la quantité d'énergie lumineuse absorbée peut se faire par la modifi- cation de la composition en pigments, qui constitue un mécanisme majeur d'acclimatation au froid (Fryer et al., 1995) : production $d^{\prime}$ anthocyanes, modification du ratio entre chlorophylle $a$ et $b$ mais surtout de la proportion en caroténoïdes ( $\beta$-carotène, lutéine et xanthophylles) qui jouent un rôle important dans la dissipation de l'excès d'énergie lumineuse sous forme de chaleur (Verheul et al., 1995). Au niveau du cycle des xanthophylles, la zéaxanthine est principalement impliquée dans la dissipation thermique de l'énergie en permettant de désexciter les chlorophylles $a$ (Montané et al., 1999; Demmig-Adams et Adams, 1996). D'autres mécanismes permettent également de limiter les risques de photodommages à basse température, comme le transport cyclique des électrons et la synthèse de molécules antioxydantes (comme le glutathion) impliquées dans la destruction des ROS.

\section{Impact des basses températures sur la stabilité des membranes cellulaires et l'état hydrique des tissus}

Les températures basses induisent une déstabilisation des membranes plasmiques (Murata et Los, 1997). La fluidité des membranes cellulaires dépend de leur composition lipidique et du degré de saturation des acides gras. Le froid a une action directe sur l'ensemble des membranes cellulaires (plus les acides gras sont saturés, plus ils seront rigidifiés par le froid). Le froid, comme la salinité ou la sécheresse, provoque également une diminution du contenu relatif en eau des cellules (Maury et al., 2000, Kacperska, 2004, Poormohammad Kiani et al. 2007a, 2007b). La déshydratation des cellules peut conduire à la perte de turgescence. Il existe des relations étroites entre ces trois événements primaires. La déstabilisation des membranes plasmiques a pour conséquence une perte d'électrolytes (dont les ions $\mathrm{Ca}^{2+}$ ) à travers la membrane plasmique qui contribue à la perte de turgescence (Campos et al., 2003) et une altération du fonctionnement des protéines membranaires comme les plastoquinones, favorisant ainsi les processus de photooxydation (Gombos et al., 1994).

Ces événements primaires de réponse au froid provoquent donc des dommages, plus ou moins sévères en fonction de l'intensité du stress, sur le fonctionnement physiologique de la plante. Ils contribuent par ailleurs à l'induction des voies de signalisation et à l'activation des gènes impliqués dans les mécanismes d'acclimatation aux basses températures (Hewezi et al., 2006). L'acclimatation au froid induit une augmentation de la con- centration en sucre intracellulaire (Guinchard et al., 1997). Ces sucres permettent de réguler la pression osmotique et de diminuer le point de congélation de la cellule (Koster et Lynch, 1992). Les sucres (saccharose, glucose, fructose) ne sont pas les seules molécules impliquées dans la régulation de l'état hydrique (Yelenosky et Guy, 1989). La proline, la glycinebétaine ou les polyols sont également des osmorégulateurs dont la synthèse est augmentée en période d'acclimatation au froid (Xin et Browse, 2000). La désaturation des lipides membranaires est également un des processus majeurs dans I'acclimatation au froid. Les acides gras insaturés, plus fluides que les acides gras saturés à basse température, permettent aux membranes de conserver leurs propriétés, en particulier celles associées au fonctionnement photosynthétique au niveau des thylakoïdes (Gombos et al., 1994 ; Wada et al., 1994).

\section{Indicateurs physiologiques pour caractériser la croissance et la tolérance des génotypes aux basses températures}

\section{Températures seuils de germination et d'élongation de I'hypocotyle}

Pour pouvoir représenter le processus de levée au champ et prédire le temps de levée, il est nécessaire d'établir les températures de base pour la germination et pour l'élongation de I'hypocotyle qui sont deux processus indépendants sur lesquels la température n'a pas le même impact (Bewley et Black, 1994). Angus et al. (1980) furent parmi les premiers à proposer un modèle (basé sur une régression linéaire) pour déterminer la réponse de la phase de levée à la température chez plus de 40 espèces. Ces travaux montrent que la $\mathrm{Tb}$ de levée présente une très grande variabilité interspécifique (de 1,4 à $14,7{ }^{\circ} \mathrm{C}$ ). La Tb de levée estimée pour le tournesol est de $7,9^{\circ} \mathrm{C}$, sans différenciation des processus de germination et d'élongation de l'hypocotyle. Chez la luzerne, des différences génotypiques ont cependant été observées pour les températures de base de la germination et d'élongation de I'hypocotyle (Brunel et al., 2009). Pour caractériser l'effet des basses températures sur la levée chez le tournesol, ce qui n'a jamais été publié à notre connaissance, il faudrait donc estimer les températures de base spécifiques à la germination et à l'élongation de l'hypocotyle pour une population présentant une diversité génétique importante. Le taux final de germination à basse tem- 
pérature est un caractère qui pourrait être également évalué. Les modèles écophysiologiques qui se sont intéressés à la représentation de cette phase de croissance ont été principalement développés à partir de sousmodèles décrivant chacun un processus physiologique en fonction de la température (Blacklow, 1972 ; Wanjura et al., 1970). La relation entre la vitesse de germination et la température a souvent été décrite par un modèle linéaire (Moot et al., 2000 ; Steinmaus et al., 2000). La vitesse d'élongation de l'hypocotyle a également été décrite par une fonction linéaire du temps (Hatfield et Egli, 1974; Wheeler et Ellis, 1991). La régression linéaire présente l'avantage de pouvoir déterminer facilement un paramètre clé souvent discuté dans la littérature, la « Tb » (de germination et d'élongation de I'hypocotyle). La $\mathrm{Tb}$ (en ${ }^{\circ} \mathrm{C}$ ) se calcule à partir des deux paramètres $b_{0}$ et $b_{1}$ de la régression linéaire décrite par l'équation 1 où $v$ est la vitesse (en $j^{-1}$ ) du processus physiologique analysé (germination ou élongation) et $T$ la température (en ${ }^{\circ} \mathrm{C}$ ) (Angus et al., 1980) :

$$
v=b_{0}+b_{1} T \text { (équation 1) et } T b=\frac{-b_{0}}{b_{1}}
$$

\section{Paramètres photosynthétiques relatifs à la croissance à basse température}

L'effet des basses températures sur le fonctionnement photosynthétique peut être analysé à partir de la technique de fluorescence chlorophyllienne. À partir de différentes mesures d'émission de la fluorescence chlorophyllienne déterminées dans des conditions d'éclairement particulières, plusieurs paramètres relatifs à la phase photochimique de la photosynthèse peuvent être déterminés. L'efficience photochimique potentielle des photosystèmes II (ФP), le rendement quantique du transport non cyclique des électrons sous éclairement (TPSII), les quenchings photochimique $(\mathrm{q} P)$ et non photochimique (NPQ) sont des paramètres largement utilisés dans la littérature comme indicateurs de tolérance au froid (Fracheboud et al., 2004 ; Groom et Baker, 1992 ; Krause, 1988 ; Lee et al., 2002 ; Verheul et al., 1995). Une réduction permanente de ФP permet de mettre en évidence les dommages causés par la photo-inhibition (Maury et al., 1996 ; Gouallec et al., 1991 ; Jompuk et al., 2005) et la mesure du ФPSII permet d'évaluer la réduction de l'activité photosynthétique liée aux basses températures (Fracheboud et al., 2004 ; Fryer et al., 1998). Des zones chromosomiques impliquées dans le contrôle de ces paramètres de fluorescence ont été identifiées chez le tournesol (Poormohammad Kiani et al., 2008).
La modification de la composition en pigments observée en réponse aux basses températures est caractérisée par une diminution de la quantité de chlorophylles au profit des caroténoïdes. Elle peut être directement évaluée par dosage (Fracheboud et al., 2004 ; Leipner et al., 1999; Verheul et al., 1995), mais peut également être estimée par une mesure simple, rapide et non destructive: I'indice SPAD à I'aide d'un chlorophyllmètre (Fracheboud et al., 2004 ; Jompuk et al., 2005 ; Lee et al., 2002). Le principe d'analyse est la mesure de la transmittance lumineuse à deux longueurs d'ondes: le rouge $(650 \mathrm{~nm})$ et le proche infrarouge $(950 \mathrm{~nm})$. En optique, la transmittance $\mathrm{T}$ d'un matériau correspond à la fraction de l'intensité lumineuse le traversant: $T=\mathrm{l} / \mathrm{lo}$ où lo est l'intensité incidente et I est l'intensité sortante.

\section{Indicateurs de tolérance au froid: potentiel osmotique et stabilité des membranes cellulaires}

L'accumulation de solutés intracellulaires liée au processus d'acclimatation au froid peut être mesurée de façon directe en réalisant un dosage des sucres solubles ou d'autres osmolytes comme les ions inorganiques et métabolites tels que la glycine-betaïne et la proline (Hekneby et al., 2006; Koster et Lynch, 1992). Le potentiel osmotique foliaire est une mesure plus globale qui permet de révéler des modifications de concentration en solutés intracellulaires en réponse aux basses températures (Guinchard et al., 1997). La mesure du potentiel osmotique réalisée après réhydratation de la plante permet de déterminer l'ajustement osmotique (accumulation active de solutés) réalisé par la plante en période d'acclimatation au froid (Yelenosky et Guy, 1989).

La perte de stabilité des membranes cellulaires, induite par les basses températures, se traduit par une fuite du contenu intracellulaire vers le milieu extracellulaire. Cette perte d'électrolytes peut être estimée en mesurant la conductivité d'une solution dans laquelle une feuille a été immergée (la conductivité électrique traduit la capacité d'une solution aqueuse à conduire le courant électrique). La quantité $d^{\prime}$ électrolytes perdue après un traitement au froid ramenée à la quantité totale d'électrolytes contenue dans les cellules permet de calculer la perte relative d'électrolytes. Une valeur élevée de ce pourcentage traduit une forte fuite $d^{\prime}$ ions à travers les membranes donc des membranes instables, affectées par le froid. La perte relative d'électrolytes est un test couramment utilisé pour évaluer les dommages causés par le froid sur les cellules, dans le cas de températures basses positives (Campos et al., 2003 ; Janowiak et al., 2003), et également pour des températures négatives (Nunes et Smith, 2003).

\section{Conclusion}

Ces indicateurs communément utilisés pour mettre en évidence des modifications physiologiques liées à la tolérance au froid, comme les dégâts des membranes ou la capacité de croissance (taux de chlorophylle, potentiel photochimique), mais également liées aux mécanismes d'acclimatation au stress, comme le potentiel osmotique à pleine turgescence, sont actuellement en cours d'évaluation chez le tournesol. Les premiers résultats obtenus, suite à l'analyse d'une population cartographiée de lignées recombinantes, indiquent que le taux de chlorophylle apparaît comme un trait génétiquement associé à la tolérance au froid chez le tournesol (Allinne et al., 2009). Des zones chromosomiques majeures, impliquées dans le contrôle de traits physiologiques (QTLs) associés à la tolérance au froid, ont également été identifiées. Dans une perspective de modélisation dynamique de la réponse des génotypes de tournesol au semis précoce, une première étape consistera à évaluer les formalismes du modèle SUNFLO (Casadebaig, 2008) pour des semis précoces, en considérant plus spécifiquement les processus physiologiques affectés par les basses températures, puis, dans un second temps, il s'agira de compléter le paramétrage actuel du modèle pour y intégrer les différences génotypiques de réponse aux basses températures. L'analyse de processus physiologiques clés vise également à identifier des indicateurs d'évaluation de la variabilité génotypique pour la tolérance au gel chez le tournesol. Ainsi, une méthodologie de phénotypage intégrée, reposant sur un ensemble d'indicateurs physiologiques clés et sur la modélisation dynamique de la réponse des génotypes de tournesol au semis précoce, pourrait être proposée.

\section{RÉFÉRENCES}

Allen DJ, Ort DR. Impacts of chilling temperatures on photosynthesis in warm-climate plants. Trends Plant Sci $2001 ; 6$ : 36-42.

Allinne C, Maury P, Sarrafi A, Grieu P. Genetic control of physiological traits associated to low temperature growth in sunflower under early sowing conditions. Plant Sci 2009 ; 177 : 349-59.

Alvarado V, Bradford KJ. A hydrothermal time model explains the cardinal temperatures for seed germination. Plant Cell Environ 2002 ; 25 : 1061-9.

Angus JF, Cunningham RB, Moncur MW, Mackenzie DH. Phasic development in field crops I. Thermal response in the seedling phase. Field Crop Res 1980 ; $3: 365-78$. 
Benech-Arnold RL, Sanchez RA. Modeling weed seed germination. In : J Kigel, G Galili, eds. Seed development and germination. 1995.

Bewley D, Black M. Seeds: physiology of development and germination. New York, London : Plenum Press, 1994.

Blacklow WM. Influence of temperature on germination and elongation of the radicle and shoot of corn (Zea mays L.). Crop Sci 1972 ; 12 : 647-50.

Brunel S, Teulat-Merah B, Wagner MH, Huguet T, Prosperi JM, Durr C. Using a model-based framework for analysing genetic diversity during germination and heterotrophic growth of Medicago truncatula. Ann Bot-London 2009 ; 103 : 1103-17.

Campos PS, Quartin V, Ramalho JC, Nunes MA. Electrolyte leakage and lipid degradation account for cold sensitivity in leaves of Coffea sp. plants. J Plant Physiol 2003 ; 160 : 283-92.

Casadebaig P. Analyse et modélisation des interactions génotypes-environnement-conduite de culture : application au tournesol (Helianthus annuus L.). Thèse de doctorat, 2008.

Côme D, Corbineau F. Physiologie végétale, tome 2, croissance et développement, chapitre 2 : semences et germination. Hermann Édition, 1998.

Demmig-Adams B, Adams III WW. The role of xanthophyll cycle carotenoids in the protection of photosynthesis. Trends Plant Sci $1996 ; 1$ : 21-6.

Durr C, Aubertot JN, Richard G, Dubrulle P, Duval Y, Boiffin J. Simple: a model for simulation of plant emergence predicting the effects of soil tillage and sowing operations. Soil Sci Soc Am / 2001; 65 : 414-23.

Ensminger I, Busch F, Huner NPA. Photostasis and cold acclimation: sensing low temperature through photosynthesis. Physiol Plant $2006 ; 126$ : 28-44.

Fracheboud Y, Jompuk C, Ribaut JM, Stamp P, Leipner J. Genetic analysis of cold-tolerance of photosynthesis in maize. Plant Mol Biol $2004 ; 56$ : 241-53.

Fryer M], Oxborough K, Martin B, Ort DR, Baker NR. Factors associated with depression of photosynthetic quantum efficiency in maize at low growth temperature. Plant Physiol $1995 ; 108: 761-7$.

Fryer M], Andrews JR, Oxborough K, Blowers DA, Baker NR. Relationship between $\mathrm{CO}_{2}$ assimilation, photosynthetic electron transport, and active $\mathrm{O}_{2}$ metabolism in leaves of maize in the field during periods of low temperature. Plant Physiol $1998 ; 116$ : 571-80.

Gombos Z, Wada H, Murata N. The recovery of photosynthesis from low-temperature photoinhibition is accelerated by the unsaturation of membrane lipids: a mechanism of chilling tolerance. P Natl Acad Sci USA 1994 ; 91 : 8787-91.

Gouallec JL, Cornic G, Briantais JM. Chlorophyll fluorescence and photoinhibition in a tropical rainforest understory plant. Photosynth Res $1991 ; 27$ : $135-42$.
Groom QJ, Baker NR. Analysis of light-induced depressions of photosynthesis in leaves of a wheat crop during the winter. Plant Physiol 1992; 100 : 1217-23.

Guinchard MP, Robin C, Grieu P, Guckert A. Cold acclimation in white clover subjected to chilling and frost: changes in water and carbohydrates status. Eur J Agron 1997 ; 6 : 225-33.

Hatfield JL, Egli DB. Effect of temperature on the rate of soybean hypocotyl elongation and field rmergence. Crop Sci 1974 ; 14 : 423-6.

Hekneby M, Antolín MC, Sánchez-Díaz M. Frost resistance and biochemical changes during cold acclimation in different annual legumes. Environ Exp Bot 2006 ; 55 : 305-14.

Hewezi T, Leger M, El Kayal W, Gentzbittel L. Transcriptional profiling of sunflower plants growing under low temperatures reveals an extensive down regulation of gene expression associated with chilling sensitivity. J Exp Bot 2006 ; 57 : 3109-22.

Janowiak F, Luck E, Dörffling K. Chilling tolerance of Maize seedling in the field during cold periods in spring is related to chilling-induced increase in abscisic acid level. J Agron Crop Sci 2003; 189: 156-61.

Jompuk C, Fracheboud Y, Stamp P, Leipner J. Mapping of quantitative trait loci associated with chilling tolerance in maize (Zea mays L.) seedlings grown under field conditions. J Exp Bot 2005; 56 : 1153-63.

Kacperska A. Sensor types in signal transduction pathways in plant cells responding to abiotic stressors: do they depend on stress intensity? Physiol Plant $2004 ; 122$ : 159-68.

Koster KL, Lynch DV. Solute accumulation and compartmentation during the cold acclimation of puma rye. Plant Physiol $1992 ; 98: 108-13$.

Krause GH. Photoinhibition of photosynthesis. An evaluation of damaging and protective mechanisms. Physiol Plant $1988 ; 74: 566-74$.

Lee EA, Staebler MA, Tollenaar M. Genetic variation in physiological discriminators for cold toleranceearly autotrophic phase of maize development. Crop Sci $2002 ; 42$ : 1919-29.

Leipner J, Fracheboud Y, Stamp P. Effect of growing season on the photosynthetic apparatus and leaf antioxidative defenses in two maize genotypes of different chilling tolerance. Environ Exp Bot 1999; 42 : 129-39.

Maury P, Berger M, Mojayad F, Planchon C. Photochemical response to drought acclimation in two sunflower genotypes. Physiol Plant 1996; 98 : 57-66.

Maury P, Berger M, Mojayad F, Planchon C. Leaf water characteristics and drought acclimation in sunflower genotypes. Plant Soil 2000; 223: 153-60.
Montané MH, Petzold B, Kloppstech K. Formation of early-light-inducible-protein complexes and status of xanthophyll levels under high light and cold stress in barley (Hordeum vulgare L.). Planta 1999; 208 : 519-27.

Moot DJ, Scott WR, Roy AM, Nicholls AC. Base temperature and thermal time requirements for germination and emergence of temperate pasture species. New Zeal J Agr Res $2000 ; 43$ : 15-52.

Murata N, Los DA. Membrane fluidity and temperature perception. Plant Physiol 1997 ; 115 : 875-9.

Nunes ME, Smith GR. Electrolyte leakage assay capable of quantifying freezing resistance in rose clover. Crop Sci $2003 ; 43$ : 1349-57.

Poormohammad Kiani S, Maury P, Grieu P, Sarrafi A. QTL analysis of chlorophyll fluorescence parameters in sunflower (Helianthus annuus L.) under wellwatered and water-stressed conditions. Plant Sci $2008 ; 175:$ 565-73.

Poormohammad Kiani S, Talia P, Maury P, et al. Genetic analysis of plant water status and osmotic adjustment in recombinant inbred lines of sunflower under two water treatments. Plant Sci 2007a; 172 : 773-87.

Poormohammad Kiani S, Grieu P, Maury P, Hewezi T, Gentzbittel L, Sarrafi A. Genetic variability for physiological traits under drought conditions and differential expression of water stress-associated genes in sunflower (Helianthus annuus L.). Theor Appl Genet 2007b ; 114 : 193-207.

Steinmaus SJ, Prather TS, Holt JS. Estimation of base temperatures for nine weed species. J Exp Bot 2000 ; $51: 275-86$.

Verheul MJ, Van Hassel PR, Stamp P. Comparison of maize inbred lines differing in low temperature tolerance: effect of acclimation at suboptimal temperature on chloroplast functioning. Ann Bot-London $1995 ; 76: 7-14$.

Wada H, Gombos Z, Murata N. Contribution of membrane lipids to the ability of the photosynthetic machinery to tolerate temperature stress. Proc Natl Acad Sci USA 1994 ; 91 : 4273-7.

Wanjura DF, Buxton DR, Stapleton HN. A temperature model for predicting initial cotton emergence. Agron / $1970 ; 62$ : 741-3.

Wheeler TR, Ellis RH. Seed quality, cotyledon elongation at suboptimal temperatures, and the yield of onion. Seed Sci Res $1991 ; 1$ : 57-67.

Xin Z, Browse J. Cold comfort farm: the acclimation of plants to freezing temperatures. Plant Cell Environ $2000 ; 23: 893-902$.

Yelenosky G, Guy CL. Freezing yolerance of citrus, pinach, and petunia leaf tissue: osmotic adjustment and sensitivity to freeze induced cellular dehydration. Plant Physiol 1989 ; 89 : 444-51. 